\title{
Reação postural de jovens e idosos ativós em teste de perturbação do equilíbrio
}

\section{Postural reaction of young and actives elderly in balance perturbation test}

Priscila Gonçalves Franco ${ }^{1}$

Karini Borges dos Santos

André L. F. Rodacki1

\section{RESUMO}

O objetivo deste estudo foi comparar o desempenho de jovens e idosos ativos em teste de perturbação ântero-posterior do equilíbrio. Em adição reprodutibilidade do teste de perturbação do equilíbrio foi testada. 21 jovens $(24,6 \pm 3,9$ anos; $64,5 \pm 9,5 \mathrm{Kg} ; 169,1 \pm 9,4 \mathrm{~cm})$ e 21 idosos ativos $(66,9 \pm 5,5$ anos; $65,3 \pm 13,7 \mathrm{Kg} ; 156,1 \pm 8,3 \mathrm{~cm})$ foram avaliados e reavaliados em um teste de perturbação do equilíbrio (liberação de um peso sobre uma bandeja acoplada a palma das mãos dos participantes) em uma plataforma de força. Os resultados demonstram que o teste de perturbação do equilíbrio é reprodutível para deslocamento anterior do CoP (Centro de Pressão), mas não para velocidade de deslocamento. Os idosos apresentaram maior deslocamento $(15,6 \%)$ e velocidade anterior do centro de pressão $(236,5 \%)$ em distúrbio do equilíbrio do que seus pares jovens ( $\mathrm{p} \leq 0,05)$. Em conclusão, as reações posturais após perturbação do equilíbrio de jovens e idosos ativos são diferenciadas e as respostas de idosos parecem resultar em maior descontrole postural e consequente aumento de risco de quedas.

\section{PALAVRAS-CHAVE}

Envelhecimento; Distúrbio; Risco de quedas.

\begin{abstract}
The aim of this study was compare the performance of young and actives elderly in anterio-posterior balance perturbation test. 21 young $(24.6 \pm 3.9$ years; $64.5 \pm 9.5 \mathrm{Kg} ; 169.1 \pm 9.4 \mathrm{~cm})$ and 21 physically active elderly $(66.9 \pm 5.5$ years; $65.3 \pm 13.7 \mathrm{Kg} ; 156.1 \pm 8.3 \mathrm{~cm})$ were assessed in a balance perturbation test. The results demonstrate that balance perturbation test is reproducible for anterior displacement of the CoP (Center of Pression), but not for velocity. The elderly presented greater anterior displacement and velocity of the centre of pressure in the perturbation test than their young counterparts. In Conclusion the postural reactions after balance perturbation of young and active elderly are different and elderly's responses seem to result in less postural control and consequent increased risk of falls.
\end{abstract}

\section{KEYWORDS}

Aging; Disturbance; Risk of Falls.
Rev Bras Ativ Fis Saúde p. 178-185 DOI

http://dx.doi.org/10.12820/rbafs.v.19n1p178

1 Universidade Federal do Paraná. Programa de Pós-Graduação em Educação Física. Centro de Estudo do comportamento Motor 


\section{INTRODUÇÃO}

O crescente número de idosos em nossa sociedade, reflexo do aumento da expectativa de vida e dos avanços com cuidados relacionados à saúde, tem impulsionado preocupações particulares com a qualidade de vida desta população ${ }^{1}$.

O processo de envelhecimento é caracterizado por uma série de alterações que conduzem a perda da funcionalidade e de independência ${ }^{2}$. Entre os fatores que alteram com o decorrer da idade, a deterioração na função dos sistemas somatosensorial e motor, tem sido apresentada como variável de interesse de estudo devido relação que delineia com um equilíbrio estático pobre entre os idosos ${ }^{3}$. Assim, distúrbios - entendidos como perturbações sobre o equilíbrio - constituem uma importante forma de detectar as respostas do sistema de controle postural, as quais são relevantes para identificar estratégias empregadas para evitar quedas.

As quedas constituem um grave problema de saúde ${ }^{4}$ e são as maiores responsáveis por fraturas no quadril ${ }^{5}$ e morbidez entre os idosos ${ }^{6}$. Na maioria das vezes as quedas ocorrem durante a marcha e normalmente são ocasionadas por um tropeço ${ }^{7-9}$. Portanto, manutenção da estabilização e capacidade de neutralizar desequilíbrio (controle postural reativo) são fatores essenciais para vida independente ${ }^{3}$.

Estudos que se dedicam a análise de controle postural e risco de queda, geralmente utilizam em suas mensurações testes com parâmetros de equilíbrio estático, realizados em posição ortostática e podem não detectar tais déficits em idosos ${ }^{10}$. Assim, testes de perturbação do equilíbrio parecem ser mais funcionais para a avaliação do risco de quedas em idosos por melhor representarem a realidade. $\mathrm{O}$ presente estudo tem como objetivo investigar a resposta postural de idosos ativos durante perturbações ântero-posteriores do equilíbrio e comparar com o desempenho de jovens. Informações sobre capacidade de reação mediante um desequilíbrio em idosos podem auxiliar no desenvolvimento de estratégias de intervenção que possibilitem reduzir o risco de quedas nessa população. Em adição, o estudo visou testar a reprodutibilidade do teste de perturbação do equilíbrio entre idosos ativos, visto que avaliações dinâmicas possuem maior especificidade em relação às condições em que distúrbios do equilíbrio ocorrem nas situações do cotidiano.

\section{MÉTODOS}

A amostra foi composta por 42 sujeitos de ambos os gêneros, sendo 21 jovens saudáveis $(24.6 \pm 3.9$ anos; $64.5 \pm 9.5 \mathrm{Kg} ; 169.1 \pm 9.4 \mathrm{~cm})$ e 21 idosos ativos $(66.9 \pm 5.5$ anos; $65.3 \pm 13.7 \mathrm{Kg} ; 156.1 \pm 8.3 \mathrm{~cm})$, com capacidade de realizar independentemente as atividades da vida diária informadas por auto relato. Sujeitos que não aceitaram participar do estudo, apresentaram contraindicações absolutas ou relativas para as quais o protocolo de testes propostos não foi recomendável, não foram incluídos no estudo. Todos participantes assinaram termo de consentimento livre e esclarecido e os procedimentos experimentais do estudo foram aprovados pelo conselho de ética de pesquisa da UFPR.

Os participantes foram solicitados a comparecer em duas sessões no laboratório com um intervalo de 48 horas para realizar o teste e reteste de pertur- 
bação do equilíbrio (PE - P2 x P2 testes).

O experimento foi conduzido com os participantes posicionados em pé sobre uma plataforma de força (AMTI, modelo OR6-7, USA). Os sujeitos foram instruídos a permanecer com o olhar fixo em um ponto localizado a uma distância de aproximadamente $2 \mathrm{~m}$ do teste, pés descalços mantidos em posição confortável, cotovelos flexionados a $90^{\circ}$ e imobilizados por uma estrutura rígida e indeformável para impedir possíveis estratégias de membros superiores para a manutenção do equilíbrio. Sobre a palma das mãos foi acoplado uma bandeja especialmente construída para esse experimento $(0.5 \times 0.3 \mathrm{~m}$ e massa $0,3 \mathrm{Kg}$ ) sobre a qual foi subitamente liberado, por um auxiliar do estudo, um peso com altura aproximada de 25 centímetros, correspondente a $3 \%$ do peso corporal do participante. Estudo piloto apontou que cargas acima de 3\% causavam perturbações que não eram bem suportadas por idosos. A Figura 1 ilustra esquematicamente o teste de PE. Para reduzir respostas antecipatórias, o peso e a bandeja foram cobertos por um tecido escuro (não incluído na Figura 1) que não permitiu ao participante identificar o instante da liberação da carga. O teste foi realizado três vezes consecutivas em cada sujeito (PE 1, PE 2 e PE 3) com um intervalo de vinte segundos entre elas.

A magnitude do deslocamento e a velocidade média do primeiro deslocamento anterior do centro de pressão após a perturbação foram utilizadas para caracterizar a capacidade reativa à perturbação. Tais parâmetros têm sido associados com a habilidade em recuperar o equilíbrio e evitar quedas ${ }^{11}$. Os dados da plataforma de força foram exportados ao Microsoft Office Excel, onde foram determinados o instante e a posição do CoP (Centro de Pressão) no início e no final do deslocamento anterior. No gráfico do deslocamento ântero-posterior do CoP, padronizou-se como início e final do deslocamento anterior o primeiro e o último ponto da reta com maior taxa de inclinação, respectivamente (ver Figura 2).

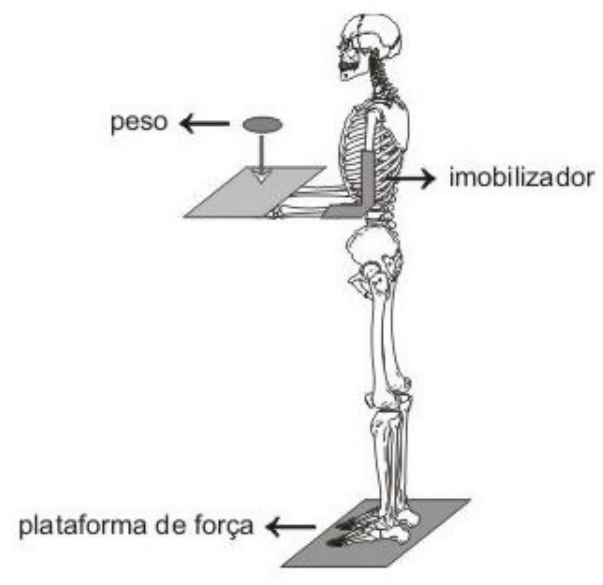

FIGURA 1 - Representação do teste de perturbação de equilíbrio

A liberação do peso provoca uma perturbação no equilíbrio do sujeito. As estratégias para recuperação do equilíbrio são registradas pela plataforma de força.

\section{Estatística}

Primeiramente os dados foram tratados através da estatística descritiva padrão (média e desvio padrão). Todas as variáveis foram submetidas ao teste de Shapiro -Wilk para confirmar a normalidade dos dados, e ao teste de Levene para confir- 
mar a homogeneidade dos grupos. Os dados que não apresentaram distribuição normal foram normalizados pela função logarítmica, quadrática ou exponencial.

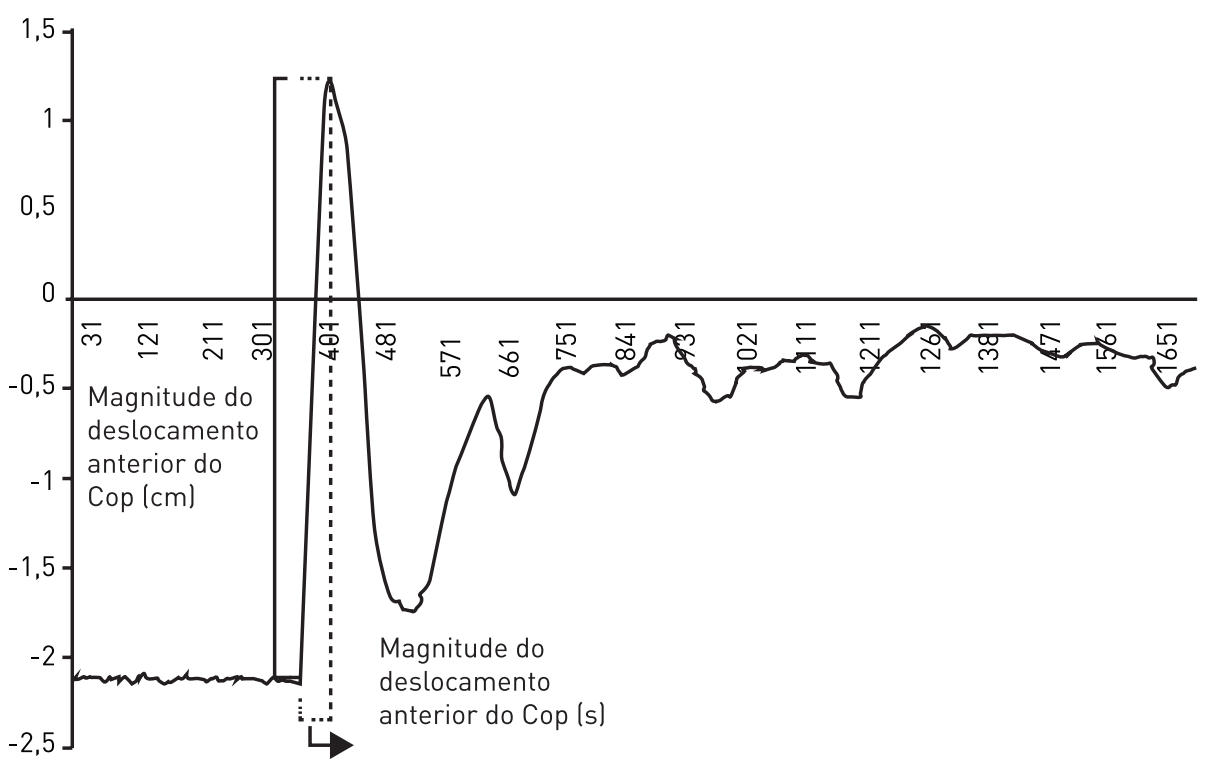

FIGURA 2 - Representação do gráfico de deslocamento ântero-posterior do CoP para teste de perturbação do equilíbrio

O teste de perturbação do equilíbrio teve sua reprodutibilidade avaliada pelo teste de Bland-Altman e foram consideradas reprodutíveis as variáveis que apresentaram variação inferior a dois desvios-padrões.

A comparação entre grupos para o teste de perturbação foi realizada através de um teste $t$ de medidas independentes. Os testes estatísticos foram realizados no software Statistica (StatSoft Inc®, versão 7.0) tendo o nível de significância aceito em $\mathrm{p} \leq 0,05$.

\section{RESULTADOS}

O teste de $\mathrm{PE}$ apresentou boa reprodutibilidade para a variável magnitude do deslocamento anterior do CoP, com variações de 4,52\% para idosos e 5,09\% para jovens. A velocidade de deslocamento anterior do CoP, apresentou elevada variação e pouca reprodutibilidade( $105.5 \%$ para os idosos e 15,8\% para os jovens) e devem ser avaliados com ponderação. A variação desses resultados está ilustrada na Figura 3.

O teste de perturbação do equilíbrio foi realizado três vezes consecutivas com cada participante (PE 1, PE 2 e PE 3). Para o PE 1, idosos apresentaram maior deslocamento anterior do CoP do que os jovens, no PE 2, além de apresentarem maior deslocamento anterior, os idosos apresentaram maior velocidade de deslocamento anterior do $\mathrm{CoP}$ do que os jovens $(\mathrm{p} \leq 0,05)$ e no $\mathrm{PE} 3$ não foram encontradas diferenças entre os grupos $(\mathrm{p}>0,05)$. O teste PE 2 foi considerado para efeitos de análise pois permite reduzir a influência da novidade e da aprendizagem que pode ter influenciado o primeiro (PE 1) e o último teste (PE 3). A Tabela 1 apresenta os resultados das 3 execuções do teste e a diferença percentual entre os grupos. 

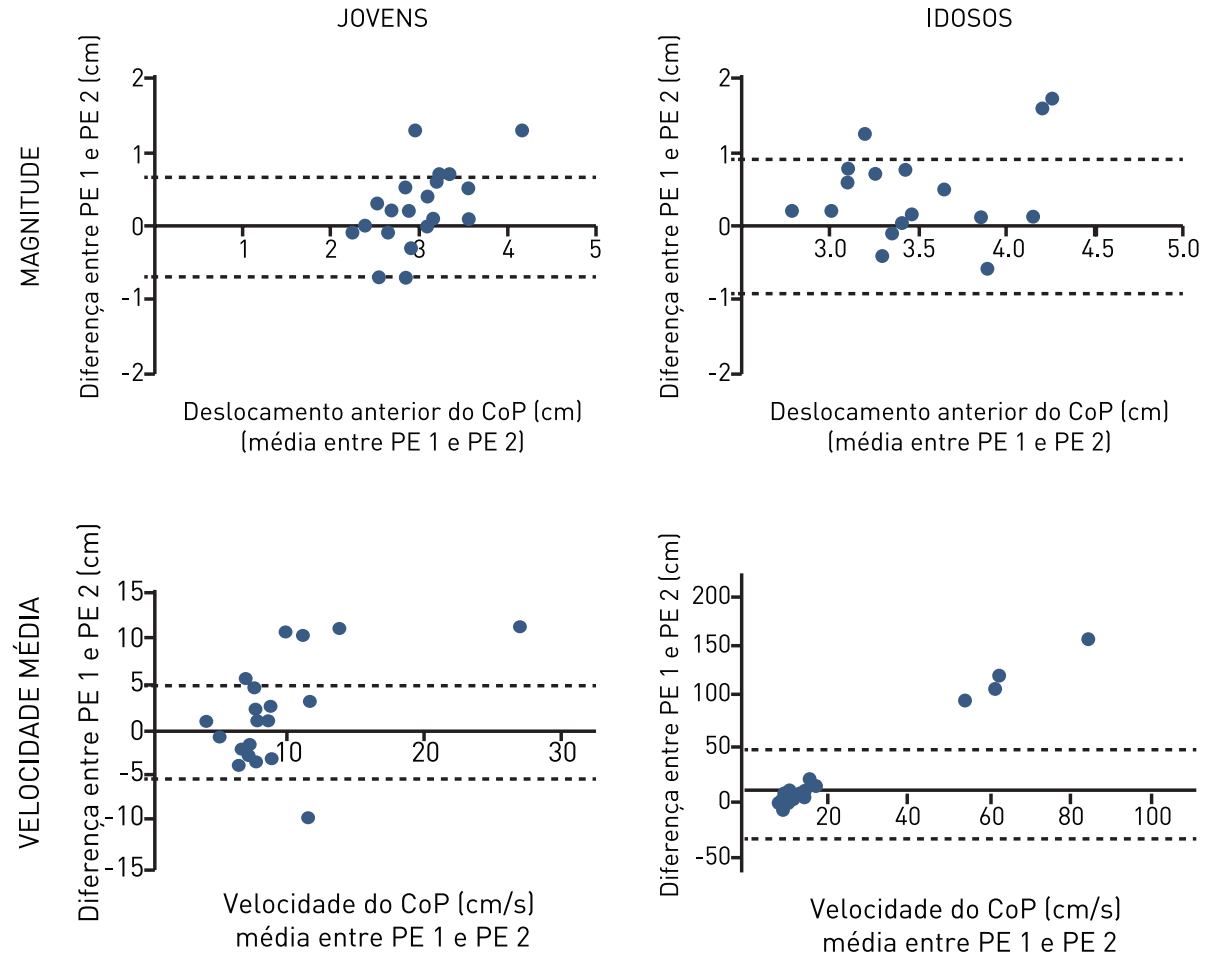

FIGURA 3 - Magnitude e velocidade média do deslocamento anterior do CoP no teste de PE realizado em intervalo de 48 horas (PE teste e PE reteste) para jovens (coluna esquerda) e idosos (coluna direita).

TABELA 1 - Teste de perturbação do equilíbrio - diferença entre idosos e jovens

\begin{tabular}{llccrc}
\hline & & Idosos (N=21) & Jovens $(\mathrm{N}=21)$ & Diferença $(\%)$ & $\mathrm{P}$ \\
\hline P1 & Deslocamento $(\mathrm{cm})$ & $4,5 \pm 1,25$ & $3,31 \pm 0,78$ & $22,36^{*}$ & 0,02 \\
\cline { 2 - 6 } & Velocidade média $(\mathrm{cm} / \mathrm{s})$ & $11,36 \pm 8,56$ & $9,87 \pm 5,55$ & 15,10 & 0,51 \\
\hline \multirow{2}{*}{ P2 } & Deslocamento $(\mathrm{cm})$ & $3,56 \pm 0,73$ & $3,08 \pm 0,67$ & $15,58^{*}$ & 0,03 \\
\cline { 2 - 5 } & Velocidade média $(\mathrm{cm} / \mathrm{s})$ & $33,62 \pm 26,10$ & $9,99 \pm 6,52$ & $236,54^{*}$ & 0,00 \\
\hline \multirow{2}{*}{ P3 } & Deslocamento $(\mathrm{cm})$ & $3,34 \pm 0,75$ & $2,97 \pm 0,62$ & 12,46 & 0,09 \\
\cline { 2 - 5 } & Velocidade média $(\mathrm{cm} / \mathrm{s})$ & $10,62 \pm 5,23$ & $9,04 \pm 5,25$ & 17,48 & 0,33 \\
\hline
\end{tabular}

Nota: ${ }^{*} p \leqslant 0,05$

\section{DISCUSSÃO}

O objetivo deste estudo foi comparar o desempenho de jovens e idosos ativos em equilíbrio ântero-posterior, além de verificar reprodutibilidade do teste de perturbação do equilíbrio. O principal achado do estudo foi que idosos apresentam diferenças entre a magnitude e a velocidade de deslocamento do centro de pressão ântero-posterior após a perturbação do equilíbrio quando comparados com grupo de jovens.

Devido às degenerações decorrentes do envelhecimento, idosos apresentam diminuição do controle postural e consequente aumento do risco de quedas ${ }^{12-14}$. Assim, é crescente o número de estudos específicos que empregam condições dinâmicas a fim de determinar a capacidade de recuperar o controle postural após uma perturbação $0^{15,7,16,17}$. Esses testes apresentam diferentes metodologias e alguns apresentam limitações em função da alta complexidade da tarefa, elevado risco de quedas ou alto custo do aparato utilizado. O teste utili- 
zado no presente estudo buscou simular o deslocamento do centro de pressão após uma pequena perturbação, através de uma metodologia prática, simples e que não oferece riscos aos participantes.

A magnitude do deslocamento anterior do CoP no teste de PE foi considerada reprodutível tanto para idosos como para jovens. Por outro lado, a velocidade do deslocamento anterior apresentou elevada variação e, portanto, não apresentou boa reprodutibilidade. Logo, os resultados da velocidade de deslocamento do CoP devem ser analisados com cautela. O deslocamento do CoP pode ter sido mais estável por ser influenciado predominantemente pela magnitude da perturbação, que foi constante em todas as execuções do teste (3\% da massa corporal do sujeito). Cargas maiores poderiam gerar perturbações mais significativas, porém poderiam levar a quedas.

A velocidade do deslocamento é influenciada pela rapidez em que o sujeito percebe e reage à perturbação e está mais sujeita a variações perceptivas. Antecipação ou tentativas em controlar a resposta à perturbação podem ter aumentado à variabilidade dos resultados. Déficits de atenção e perda de concentração decorrentes do envelhecimento ${ }^{14}$, também podem justificar a elevada variabilidade encontrada no grupo de idosos, ainda que a duração dos testes e o tempo de intervalo tenham sido reduzidos ao máximo.

$\mathrm{O}$ maior deslocamento anterior do $\mathrm{CoP}$ em idosos pode ser explicado em partes por possíveis diferenças na capacidade contrátil (habilidade de gerar torque) entre jovens e idosos. Além disso, a menor capacidade de percepção e reação em idosos ${ }^{14}$ também pode ter influenciado o tempo de resposta. Alterações posturais, quase sempre presentes em idosos, também podem ter contribuído visto que tendem a aumentar o deslocamento do centro de massa que é projetado à frente, tornando-os mais propensos a desequilíbrios anteriores ${ }^{18}$. A influência da menor velocidade de ativação muscular em idosos ${ }^{19}$ sobre o deslocamento e o tempo de reversão do centro de massa após uma perturbação parece ter sido mínima, uma vez que os aspectos temporais da ativação muscular podem desempenhar um papel menor no controle do deslocamento excessivo do centro de massa após perturbações ${ }^{20}$. Diferenças na carga aplicada não explicam as diferenças entre jovens e idosos, posto que a carga foi proporcional à massa corporal dos participantes ${ }^{15}$.

Idosos apresentaram maior velocidade no deslocamento anterior do CoP quando comparados aos jovens. A velocidade de ativação muscular após a perturbação pode ter influenciado a velocidade de deslocamento anterior do centro de pressão. Quanto mais rápido os sujeitos iniciam a ativação muscular e atingem o pico de ativação, maior é a frenagem do movimento e maiores as chances de reverter o deslocamento causado pela perturbação a fim de evitar uma queda ${ }^{21,22}$. É conhecido que idosos apresentam um atraso na ativação muscular, além de reduzida força e massa muscular ${ }^{20,15,3}$. Esses três fatores podem explicar o maior retardo para interromper a aceleração do deslocamento anterior do CoP e o maior desequilíbrio ântero-posterior dos idosos.

Esta pesquisa não incluiu análise eletromiográfica e cinemática sobre o teste de perturbação do equilíbrio o que pode dificultar um entendimento mais aprofundado sobre as respostas posturais entre jovens e idosos ativos e compreendem uma limitação do estudo. Trabalhos futuros são incentivados com inclusão de grupos sedentários para melhor entendimento sobre a influência do nível de atividade física na reação postural perante uma per- 
turbação, assim como, identificação de intervenção capaz de desenvolver ou conservar respostas de velocidade e deslocamento do centro de pressão, similares a de jovens que são mais adequadas para o reestabelecimento do controle postural.

\section{CONCLUSÃO}

O teste de perturbação do equilíbrio foi considerado reprodutível para deslocamento do centro de pressão para jovens e idosos ativos. Entretanto os resultados da velocidade de deslocamento do CoP apresentaram maior variação e devem ser analisados com cautela.

Os idosos apresentaram maior deslocamento e velocidade anterior do centro de pressão no teste de perturbação do equilíbrio do que seus pares jovens. Conclui-se que, as reações posturais após perturbação do equilíbrio de jovens e idosos ativos são diferenciadas e as respostas de idosos parecem resultar em maior descontrole postural e consequente aumento de risco de quedas.

\section{REFERÊNCIAS}

1. Lowry KA, Vallejo AN, Studenski SA. Successful aging as a continuum of functional independence: lessons from physical disability models of aging. Aging Dis. 2012; 3: 5-15.

2. Chou CH, Hwang CL, Wu YT. Effect of exercise on physical function, daily living activities, and quality of life in the frail older adults: a meta-analysis. Arch Phys Med Rehabil. 2012; 93: 237-44.

3. Lin SI, Woollacott M. Association between sensorimotor function and functional and active balance control in the elderly. Age Ageing. 2005; 34: 358-63.

4. Zheng J, Pan Y, Hua Y, Shen H, Wang X, Zhang Y, Fan Y, Yu Z. Strategic targeted exercise for preventing falls in elderly people. J Int Med Res. 2013; 41: 418-26.

5. Prince F, Corriveau H, Hébert R, Winter DA. Gait in elderly. Gait and Posture. Canada: Elsevier; 1997; 128-35.

6. Bento PCB, Rodacki ALF, Homann DE, Leite N. Exercícios físicos e redução de quedas em idosos: uma revisão sistemática. Rev Bras Cineantropom Desempenho Humano. 2010; 12: 470-78.

7. Pijnappels M, Reeves ND, Maganaris CN, Dieën JHV. Tripping without falling; lower limb strength, a limitation for balance recovery and a target for training in the elderly. J of Electromyogr Kinesiol. 2008; 18: 188-96.

8. Barak Y, Wagenaar RC, Holt KG. Gait characteristics of elderly people with a history of falls: a dynamic approach. Phys Ther. 2006; 86: 1501- 510.

9. Borgert AJ, Pavol MJ, Grabiner MD. Response time is more important than walking speed for the ability of older adults to avoid a fall after a trip. J Biomech. 2002; 35: 199-205.

10. Melzer I, Benjuya N, Kaplanski, J. Postural stability in the elderly: a comparison between fallers and non-fallers. Age Ageing. 2004; 33: 602-06.

11. Melzer I, Elbar O, Tsedek I, Oddsson L. A water-based training program that include perturbation exercises to improve stepping responses in older adults: study protocol for a randomized controlled cross-over trial. BMC Geriatrics. 2008; 8.

12. Krause MP, Buzzachera CF, Hallage T, Santos ECR, Silva SG. Alterações morfológicas relacionadas à idade em mulheres idosas. Rev Bras Cineantropom Desempenho Humano. 2006; 8: 73-77.

13. Resende SM, Rassi CM, Viana FP. Efeitos da hidroterapia na recuperação do equilíbrio e prevenção de quedas em idosas. Rev Bras Fisioter. 2008; 12: 57-63.

14. Woollacott M, Shumway-Cook A. Attention and the control of posture and gait: a review of an emerging area of research. Gait Posture. 2002; 16: 1-14.

15. Lee HJ, Chou LS. Detection of gait instability using the center of mass and center of pressure inclination angles. Arch Phys Med Rehabil. 2006; 87: 569-75. 
16. Jensen JL, Brown LA, Woollacott MH. Compensatory stepping: the biomechanics of a preferred response among older adults. Exp Aging Res. 2001; 27: 361-76.

17. Brauer SG, Neros C, Woollacott M. Balance control in the elderly: do Masters athletes show more efficient balance responses than healthy older adults? Aging Clin Exp Res. 2008; 20: 406-11.

18. Qu X, Nussbaum MA, Madigan ML. Model-based assessments of the effects of age and ankle fatigue on the control of upright posture in humans. Gait Posture. 2009; 30: 518-22.

19. Thelen DG, Muriuki M, James J, Schultz AB, Ashton-Miller JA, Alexander NB. Muscle activities used by young and old adults when stepping to regain balance during a forward fall. J Electromyogr Kinesiol. 2000; 10: 93-101.

20. Freitas PB, Knight CA, Barela JA. Postural reactions following forward platform perturbation in young, middle-age, and old adults. J Electromyogr Kinesiol. 2010, 20: 693-700.

21. Gu MJ, Schultz AB, Shepard NT, Alexander NB. Postural control in young and elderly adults when stance is perturbed: dynamics. J Biomech. 1996; 29: 319-29.

22. Okada S, Hirakawa K, Yoshihiro T, Takada Y, Kinoshita H. Age-related differences in postural control in humans in response to a sudden deceleration generated by postural disturbance. Eur J Appl Physiol. 2001; 85: 10-18.

\section{ENDERECCO PARA}

CORRESPONDÊNCIA

KARINI BORGES DOS SANTOS

Travessa Iguaçu, 66, Centro

Campo Largo/PR - 83.601-125

Telefone: (41) 3360-4333

E-mail: kariniborges21agmail.com 